\title{
Whole genome re-sequencing reveals genome-wide variations among parental lines of 16 mapping populations in chickpea (Cicer arietinum L.)
}

Mahendar Thudi ${ }^{1 *+}$, Aamir W. Khan ${ }^{1+}$, Vinay Kumar ${ }^{1}$, Pooran M. Gaur ${ }^{1}$, Krishnamohan Katta $^{1}$, Vanika Garg ${ }^{1}$, Manish Roorkiwal ${ }^{1}$, Srinivasan Samineni ${ }^{1}$ and Rajeev K. Varshney ${ }^{1,2^{*}}$

From The 3rd International Conference on Plant Genetics, Genomics, Bioinformatics and Biotechnology (PlantGen2015)

Novosibirsk, Russia. 17-21 June 2015

\begin{abstract}
Background: Chickpea (Cicer arietinum L.) is the second most important grain legume cultivated by resource poor farmers in South Asia and Sub-Saharan Africa. In order to harness the untapped genetic potential available for chickpea improvement, we re-sequenced 35 chickpea genotypes representing parental lines of 16 mapping populations segregating for abiotic (drought, heat, salinity), biotic stresses (Fusarium wilt, Ascochyta blight, Botrytis grey mould, Helicoverpa armigera) and nutritionally important (protein content) traits using whole genome re-sequencing approach.

Results: A total of $192.19 \mathrm{~Gb}$ data, generated on 35 genotypes of chickpea, comprising 973.13 million reads, with an average sequencing depth of $\sim 10 \mathrm{X}$ for each line. On an average $92.18 \%$ reads from each genotype were aligned to the chickpea reference genome with $82.17 \%$ coverage. A total of 2,058,566 unique single nucleotide polymorphisms (SNPs) and 292,588 Indels were detected while comparing with the reference chickpea genome. Highest number of SNPs were identified on the Ca4 pseudomolecule. In addition, copy number variations (CNVs) such as gene deletions and duplications were identified across the chickpea parental genotypes, which were minimum in PI 489777 ( 1 gene deletion) and maximum in JG 74 (1,497). A total of 164,856 line specific variations (144,888 SNPs and 19,968 Indels) with the highest percentage were identified in coding regions in ICC 1496 (21\%) followed by ICCV 97105 (12\%). Of 539 miscellaneous variations, 339, 138 and 62 were inter-chromosomal variations (CTX), intrachromosomal variations (ITX) and inversions (INV) respectively.

Conclusion: Genome-wide SNPs, Indels, CNVs, PAVs, and miscellaneous variations identified in different mapping populations are a valuable resource in genetic research and helpful in locating genes/genomic segments responsible for economically important traits. Further, the genome-wide variations identified in the present study can be used for developing high density SNP arrays for genetics and breeding applications.
\end{abstract}

Keywords: Chickpea, Re-sequencing, Copy number variations, Mapping populations

\footnotetext{
*Correspondence: t.mahendar@cgiar.org; r.k.varshney@cgiar.org

${ }^{\dagger}$ Equal contributors

IInternational Crops Research Institute for the Semi-Arid Tropics (ICRISAT),

Hyderabad, India

Full list of author information is available at the end of the article
}

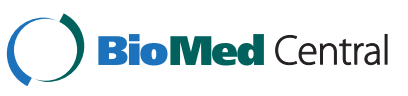

(c) 2016 Thudi et al. Open Access This article is distributed under the terms of the Creative Commons Attribution 4.0 International License (http://creativecommons.org/licenses/by/4.0/), which permits unrestricted use, distribution, and reproduction in any medium, provided you give appropriate credit to the original author(s) and the source, provide a link to the Creative Commons license, and indicate if changes were made. The Creative Commons Public Domain Dedication waiver (http://creativecommons.org/publicdomain/zero/1.0/) applies to the data made available in this article, unless otherwise stated. 


\section{Background}

Chickpea (Cicer arietinum L.) is the second most important grain legume cultivated mostly on residual soil moisture in the arid and semi-arid regions of the world. It is a self-pollinated crop and cross pollination is a rare event (0-1 \%) [1]. Chickpea has its origin in southeastern Turkey, and after its domestication, from a closely related wild species $C$. reticulatum Ladizinsky, in the Middle East this crop progressed further throughout the Mediterranean region, India and Ethiopia [2, 3]. It is a rich source of protein to vegetarian diets, especially in India. Globally, it is cultivated on over 13.5 Mha with an annual production of 13.1 million tons [4] and productivity is less than $1 \mathrm{t} / \mathrm{ha}$ which is very much less than estimated potential of 6 t/ha under optimum growing conditions. In India, it is cultivated on 9.6 Mha with 8.8 million tons production and an average productivity of $920 \mathrm{~kg} / \mathrm{ha}$. About $71 \%$ of the global area with $67 \%$ of global production of chickpea is contributed by India. Despite being the largest producer, India imports chickpea from several countries e.g. Australia, Turkey, Mexico, USA, Canada etc.

Several biotic and abiotic stress have been affecting the chickpea productivity. However, efforts to increase the productivity could not yield much success due to low genetic diversity in cultivated gene pool [5]. This limited genetic diversity in the cultivate gene pool affects genetics and genomic studies in chickpea as number of markers found polymorphic between parents were comparatively very low in comparison to other crops. For instance after screening more than two thousand markers on intra-specific chickpea populations (ICC $4958 \times$ ICC 1882 and ICC $283 \times$ ICC 8261 ) only couple of hundred representing $\sim 10 \%$ of total polymorphic markers could be identified for these populations [6]. In cases with low genetic diversity, identification of polymorphic markers between contrasting parents is time consuming and tedious task [7].

Recent advances in next-generation sequencing (NGS) technologies dramatically reduced the cost on sequencing and are being deployed to understand the genome architecture, variations in genomes, identify the candidate genes for biotic and abiotic stresses that limit crop productivity below the production potential [8]. In order to harness the untapped genetic potential available for crop improvement in a species, several germplasm lines have been re-sequenced using whole genome resequencing (WGRS) approach in different crops. For instance, 3000 rice genomes [9], maize [10], sorghum [11] etc., have been re-sequenced.

During recent years, small variations in the form of single nucleotide polymorphisms (SNPs) and Indels are being extensively deployed in crop improvement. Sometimes these small variations do not capture all the genomic information associated with a particular phenotypic variation. This may be due to other important class of large genomic variations i.e. structural variations (SVs). These SVs include inversions, translocations, transversions, copy number variations (CNVs), insertions and deletions, are genomic rearrangements ranging from 50 nucleotides to several megabases with respect to the reference genome $[12,13]$. In case of humans, these large variations are extensively studied and are associated with important complex disease phenotypes. Nevertheless, in case of plants, very few studies explored the usefulness of large variations for instance in maize, SVs have been studied between maize and its progenitor [14], while functional impact and origin mechanisms of CNVs were reported in case of barley [15].

Nevertheless, the availability of draft genome sequence for several crop plants [16] including chickpea [17], opened new vistas for crop improvement strategies. Understanding genome wide variation among parental lines of mapping populations will enable trait mapping and identification of stress responsive candidate genes. With an objective to understand the genome-wide variations that can be deployed for chickpea improvement, we resequenced a set 35 genotypes that are parental lines of 16 mapping populations and segregate for different biotic and abiotic stresses as well as nutritionally important traits in chickpea.

\section{Results and discussion}

To dissect complex biotic and abiotic stresses, several bi-parental mapping populations and next generation mapping populations like multi-parent advanced generation intercross (MAGIC) population are being used at ICRISAT. Although few thousand simple sequence repeat (SSR) markers are available for trait mapping in chickpea, limited polymorphism among parental lines of bi-parental mapping population has been hindering the trait mapping efforts to reach to the candidate genes responsible for the traits of interest [7]. Nevertheless, genome-wide variations like SNPs, CNVs and PAVs are very important for trait mapping and crop improvement and gaining importance in recent years.

In order to gain insights into the genome-wide variations that can be used for trait dissection and in-turn for chickpea improvement, 35 chickpea genotypes with diverse origin (India, Mexico, Turkey, Tanzania, Commonwealth of Independent States and Russia) and representing both market classes (desi and kabuli) were re-sequenced in this study. These 35 chickpea genotypes are parental lines of 16 mapping populations segregating for abiotic (drought, heat, salinity), biotic stresses (Fusarium wilt, Ascochyta blight, Botrytis grey mould, Helicoverpa pod borer) and nutritionally important (protein content) traits; parental lines of 
MAGIC population and parental lines of markerassisted recurrent selecion (MARS) populations (Additional file 1).

\section{In silico mapping of sequence data}

A total of $192.19 \mathrm{~Gb}$ comprising of 973.13 million 150 and $100 \mathrm{bp}$ reads were generated for 35 genotypes of chickpea at an average sequencing depth of 10.32X for each line (Additional file 2). The trimming and processing of the data resulted in 911.22 million high quality reads. On aligning the clean data, using Bowtie 2, to the CDC Frontier reference genome the mapping rate of reads varied from $90.19 \%$ (IG 72953) to $95.3 \%$ (JG 62). The variation in mapping rate among different genotypes may be due to divergence among the parental genotypes used in the study. On an average $92.18 \%$ reads from each genotype were aligned to the reference genome with $82.17 \%$ average coverage. The number of reads from each genotype aligned on to reference genome varied from 12,765,493 (ICC 1496) to 87,487,094 (JAKI 9218) while uniquely aligned reads varied from $7,778,952$ (ICC 1496) to 40,072,407 (JAKI 9218) thus on an average $53.92 \%$ high quality reads were uniquely aligned to the genome. The mean depth ranged from 5.79 to 20.04 with an average of $\sim 8.6$ for all the samples. Higher mean depths of 14.26 and 20.04 were observed in ICC 4958 and JAKI 9218 because of comparatively higher amount of reads generated for these samples (Additional file 2).

\section{SNPs and their distribution}

To determine the extent of sequence diversity among 35 chickpea genotypes, clean reads were aligned to the reference genome assembly of chickpea. As a result, a total of 2,058,566 SNPs were identified across all 35 genotypes re-sequenced (Additional file 3). Prior to this study, 51,632 SNPs were identified by 454 transcriptome sequencing of Cicer arietinum and Cicer reticulatum genotypes [18]. In addition, few hundreds of SNPs were also reported using Solexa / Illumina sequencing, amplicon sequencing of tentative orthologous genes (TOGs), mining of expressed sequence tags (ESTs) and sequencing of candidate genes [19-21]. WGRS approach has also been deployed in several crops for instance soybean [22], rice [23], pepper [24], maize [25] and tomato [26]. Among the SNPs on eight pseudomolecules (Ca1 to $\mathrm{Ca} 8$ ), most SNPs were identified on Ca4 $(377,491)$ and the least on Ca8 $(79,770)$, accounting for 18.34 and $3.88 \%$ of the SNPs, respectively (Fig. 1a; Additional file 3). A total of 361,177 SNPs were identified on unanchored scaffolds and contigs $(\mathrm{Ca} 0)$ accounting to $17.55 \%$ of SNPs identified. The SNP density varied among pseudomolecules; $\mathrm{Ca} 4$ has the highest density (7.67 SNPs per $\mathrm{Kb}$ ) and $\mathrm{Ca} 0$ had the lowest density (1.954 SNPs per Kb) (Additional file 3). Amongst the pseudomolecules, $\mathrm{Ca} 4$ was found to have maximum polymorphism rate $(8.92 / \mathrm{Kb})$, while $\mathrm{Ca} 7$ had least polymorphism rate $(3.95 / \mathrm{Kb})$. This study further re-affirms the results reported earlier [17] which may be due to presence of large repetitive regions in Ca4 pseudomolecule. The minimum density for exonic variants was observed on Ca7 (0.16 exon variants/Kb) while the maximum was found on Ca4 (0.36 exon variants $/ \mathrm{Kb}$ ) among the pseudomolecules (Additional file 3). Least density for exonic variants was 0.02 exon variants/ $\mathrm{Kb}$ on $\mathrm{Ca} 0$. This means there were maximum changes in the coding regions of $\mathrm{Ca} 4$, in concurrence with the result of Varshney et al $[17,31]$. The number of SNPs per genotype varied from 97,091 (ICCV 04516) to 1,001,744 (IG 72953) (Table 1). ICC 4958 among desi and ICC 8261 among kabuli genotypes were found to have maximum number of SNPs. The number of pair-wise SNPs were high between IG 72953 and IG 72933 (1,133,522 SNPs) and least between CDC-Frontier and ICCV 04516 $(97,091)$ (Additional file 4). The number of SNPs reported in the study are higher compared to the previous studies $[17,27-30]$. This may be due to diverse parental lines and wild genotypes used in the present study. The SNPs were categorized further into homozygous and heterozygous SNPs based on called SNPs in each genotype against the reference genome (Additional files 5 and 6). Maximum number of homozygous SNPs were identified in case of PI $489777(606,413)$ and minimum in case of ICCV 04516 (57,432 SNPs). Among 35 genotypes maximum heterozygosity rate was observed in case of IG 72933 (0.84), while least heterozygosity rate was observed in case of PI 489777 (0.08). The mean heterozygosity rate was 0.36 across the 35 genotypes (Additional file 6).

\section{Insertions and deletions (Indels)}

Insertions and deletions ranging from $1 \mathrm{bp}$ to $58 \mathrm{bp}$ were considered as Indels in the present study. In total, 292,588 Indels were identified across 35 chickpea genotypes (Additional file 3). The maximum number of deletions, 81,516 were $1 \mathrm{bp}$ in length, while the least number of deletions (2) were of 52, 53, 56 and 57 bp in lengths. The maximum number of insertions were 78,678 with 1 bp length, while the minimum number was 1 with 58 bp length (Additional file 7). Of these Indels, 148,309 were the deletions and 144,279 were insertions. The density of deletions and insertions were 0.28 and 0.27 per $\mathrm{Kb}$ respectively across the genome (Additional file 3 ). Further, Indel analysis for each sample against the reference, CDC-Frontier, revealed the maximum Indels in IG $72953(115,538)$, and minimum Indels in ICCV $04516(13,146)$ respectively (Fig. 1b). When insertions to deletions ratio was calculated for each 


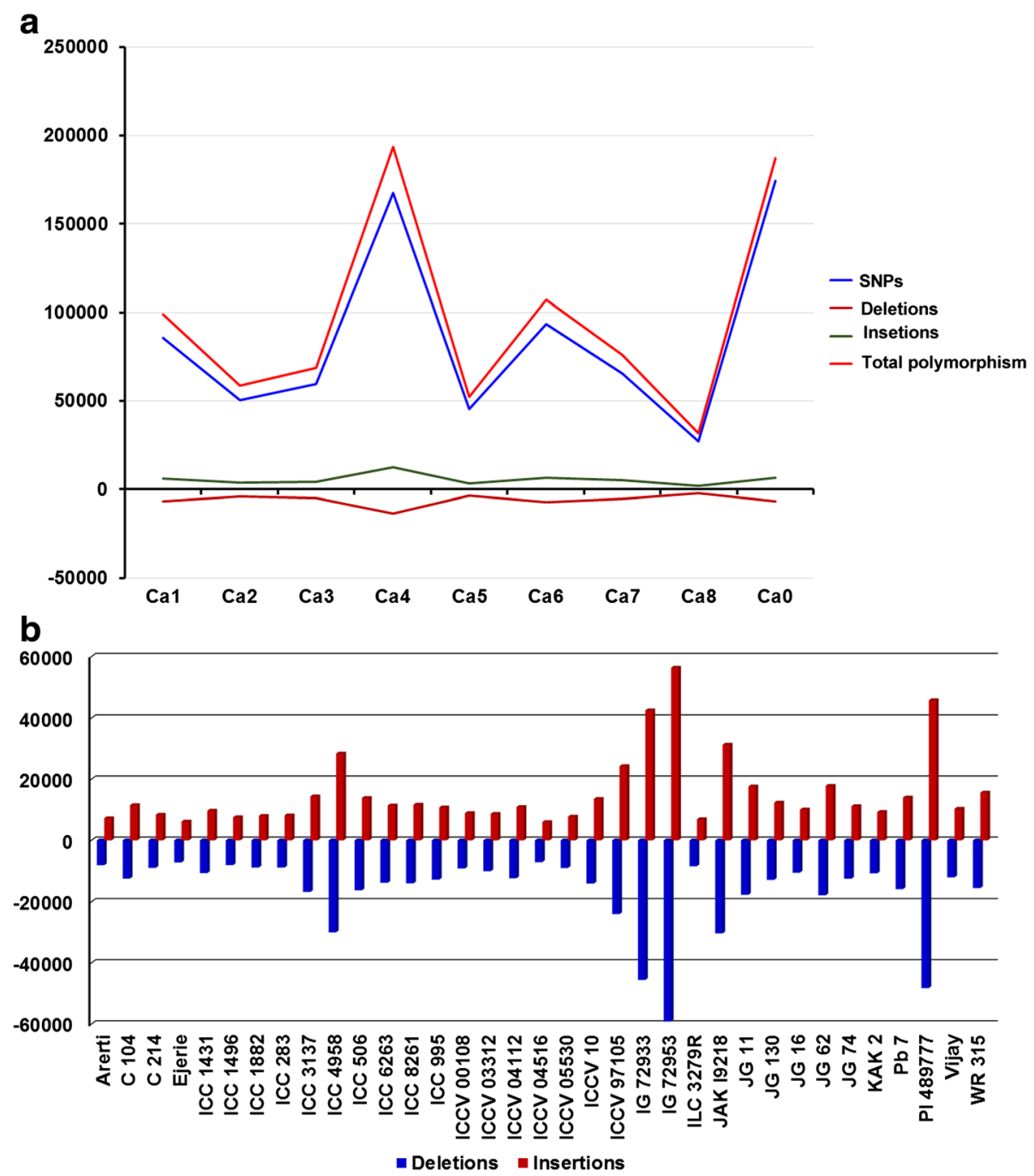

Fig. 1 Genome-wide variations in 35 chickpea genotypes. a Distribution of SNPs, insertions, deletions and polymorphisms on eight pseudomolecules of chickpea. $\mathbf{b}$ Insertions and deletions identified in each chickpea genotype used in the present study

genotype, the maximum and minimum indel ratios were 1.03 and 0.81 in case of JAKI 9218 and ILC 3279 respectively. In JG 11, JG 62, WR 315 and ICCV 97105 the Indel ratio was $\sim 1$ (Additional file 8).

\section{Copy number variations (CNVs) and presence absence variations (PAVs)}

CNVs and PAVs were determined in case of genes longer than $1 \mathrm{~Kb}$. The gene ontology analysis was done using Swiss-prot and Trembl databases (http:// www.ebi.ac.uk/uniprot). A non-redundant set of 9,732 genes were found duplicated across different genotypes and for 9,628 genes Uniprot IDs were retrieved and assigned. Out of these, 4,374 genes were found duplicated across just one of the samples making them line specific duplicated genes. Gene Ca_27299 was found duplicated across a maximum of 23 samples (Additional file 9). Ca_27299 with GO IDs GO:0016021; GO:0005886 was found to be Receptor-like protein 12 (AtRLP12) present to function for cell membrane. Duplicated genes ranged from 4 to 1135 amongst different genotypes. In JG 74 , a maximum of 1,135 genes were found duplicated while the minimum number of genes (4) were duplicated in JG 62 (Additional file 9). Maximum number of defence related genes (27) were duplicated in a salt tolerant line ICC 1431.

Similarly, a non-redundant set of 205 genes were not found in any genotype. Uniprot IDs could be assigned for 198 genes. Amongst these, 134 genes were not present in any of the genotypes suggesting line specific gene deletion (Additional file 10). The gene $\mathrm{Ca}_{-} 17015$ was absent in eight genotypes, however, it is an uncharacterized protein. Ca_13947 was not present in 7 
Table 1 Summary of inter-and intra-genic SNPs in 35 chickpea genotypes

\begin{tabular}{|c|c|c|c|c|c|c|c|c|c|c|c|}
\hline \multirow[t]{4}{*}{ Genotype } & \multirow{4}{*}{$\begin{array}{l}\text { Intergenic } \\
\text { No. of } \\
\text { intergenic }\end{array}$} & \multicolumn{8}{|c|}{ No of intragenic SNPs } & \multirow[t]{4}{*}{ Others } & \multirow[b]{4}{*}{ Total } \\
\hline & & \multirow{3}{*}{$\begin{array}{l}\text { Intron } \\
\text { No. of intronic } \\
\text { SNPs }\end{array}$} & \multicolumn{7}{|l|}{ Exon } & & \\
\hline & & & \multicolumn{5}{|l|}{ Non-synonymous } & \multicolumn{2}{|l|}{ Synonymous } & & \\
\hline & & & $\begin{array}{l}\text { No. of non-synonymous } \\
\text { coding }\end{array}$ & $\begin{array}{l}\text { No. of non-synonymous } \\
\text { start }\end{array}$ & $\begin{array}{l}\text { No. of start } \\
\text { lost }\end{array}$ & $\begin{array}{l}\text { No. of stop } \\
\text { gained }\end{array}$ & $\begin{array}{l}\text { No. of stop } \\
\text { lost }\end{array}$ & $\begin{array}{l}\text { No. of synonymous } \\
\text { coding }\end{array}$ & $\begin{array}{l}\text { No. of synonymous } \\
\text { stop }\end{array}$ & & \\
\hline Arerti & 95,463 & 11,373 & 1,836 & 1 & 2 & 23 & 10 & 1,906 & 2 & 967 & 111,583 \\
\hline C 104 & 141,435 & 18,636 & 2,887 & 2 & 2 & 31 & 8 & 3,180 & 1 & 1,194 & 167,376 \\
\hline C 214 & 111,023 & 13,121 & 2,094 & 2 & 5 & 30 & 11 & 2,221 & 2 & 902 & 129,411 \\
\hline Ejerie & 84,715 & 10,047 & 1,719 & 0 & 4 & 18 & 10 & 1,786 & 2 & 964 & 99,265 \\
\hline ICC 1431 & 126,604 & 16,306 & 2,456 & 1 & 5 & 25 & 12 & 2,828 & 3 & 1,007 & 149,247 \\
\hline ICC 1496 & 99,289 & 11,615 & 1,807 & 2 & 3 & 19 & 6 & 1,992 & 2 & 843 & 115,578 \\
\hline ICC 1882 & 107,060 & 12,517 & 1,936 & 3 & 3 & 23 & 11 & 2,101 & 5 & 882 & 124,541 \\
\hline ICC 283 & 109,146 & 13,296 & 1,943 & 1 & 3 & 24 & 10 & 2,130 & 2 & 861 & 127,416 \\
\hline ICC 3137 & 172,561 & 20,255 & 3,189 & 1 & 8 & 45 & 20 & 3,428 & 6 & 1,314 & 200,827 \\
\hline ICC 4958 & 292,686 & 35,022 & 5,159 & 3 & 13 & 58 & 19 & 5,884 & 8 & 1,951 & 340,803 \\
\hline ICC 506 & 169,681 & 19,028 & 2,977 & 4 & 7 & 32 & 15 & 3,292 & 6 & 1,358 & 196,400 \\
\hline ICC 6263 & 151,707 & 14,874 & 2,394 & 1 & 7 & 38 & 15 & 2,481 & 5 & 1,405 & 172,927 \\
\hline ICC 8261 & 151,627 & 16,987 & 2,586 & 1 & 7 & 30 & 10 & 2,802 & 4 & 1,290 & 175,344 \\
\hline ICC 995 & 146,193 & 15,647 & 2,428 & 1 & 8 & 25 & 14 & 2,748 & 3 & 1,247 & 168,314 \\
\hline ICCV 00108 & 110,493 & 11,327 & 1,442 & 2 & 3 & 19 & 6 & 1,459 & 2 & 927 & 125,680 \\
\hline ICCV 03312 & 118,283 & 12,341 & 1,996 & 0 & 5 & 30 & 10 & 2,142 & 3 & 1,123 & 135,933 \\
\hline ICCV 04112 & 133,378 & 17,305 & 2,383 & 1 & 7 & 30 & 8 & 2,712 & 4 & 1,093 & 156,921 \\
\hline ICCV 04516 & 84,366 & 8,806 & 1,385 & 2 & 3 & 20 & 7 & 1,565 & 0 & 937 & 97,091 \\
\hline ICCV 05530 & 107,685 & 12,448 & 1,942 & 1 & 7 & 31 & 13 & 2,055 & 3 & 995 & 125,180 \\
\hline ICCV 10 & 151,630 & 17,308 & 2,251 & 1 & 3 & 24 & 6 & 2,416 & 6 & 999 & 174,644 \\
\hline ICCV 97105 & 222,311 & 28,993 & 4,029 & 3 & 7 & 35 & 12 & 4,766 & 7 & 1,243 & 261,406 \\
\hline IG 72933 & 528,292 & 87,348 & 11,343 & 5 & 27 & 80 & 35 & 15,069 & 19 & 2,236 & 644,454 \\
\hline IG 72953 & 779,323 & 166,944 & 21,356 & 5 & 29 & 119 & 53 & 31,241 & 48 & 2,626 & $1,001,744$ \\
\hline ILC 3279R & 98,538 & 9,967 & 1,845 & 1 & 1 & 30 & 12 & 1,820 & 2 & 1,092 & 113,308 \\
\hline JAKI 9218 & 271,934 & 31,648 & 4,530 & 2 & 11 & 49 & 14 & 5,105 & 5 & 1,734 & 315,032 \\
\hline JG 11 & 180,028 & 21,190 & 2,559 & 2 & 5 & 27 & 7 & 2,761 & 4 & 1,202 & 207,785 \\
\hline JG 130 & 143,400 & 16,890 & 2,501 & 2 & 6 & 27 & 4 & 2,781 & 5 & 1,046 & 166,662 \\
\hline$J G 16$ & 126,104 & 15,899 & 2,238 & 2 & 5 & 27 & 11 & 2,437 & 5 & 887 & 147,615 \\
\hline JG 62 & 172,088 & 20,888 & 3,224 & 1 & 9 & 38 & 16 & 3,580 & 6 & 1,017 & 200,867 \\
\hline
\end{tabular}


Table 1 Summary of inter-and intra-genic SNPs in 35 chickpea genotypes (Continued)

\begin{tabular}{|c|c|c|c|c|c|c|c|c|c|c|c|}
\hline$J G 74$ & 146,466 & 18,427 & 2,743 & 1 & 5 & 27 & 12 & 3,147 & 6 & 1,154 & 171,988 \\
\hline KAK 2 & 125,250 & 13,638 & 2,041 & 1 & 5 & 30 & 9 & 2,253 & 3 & 1,130 & 144,360 \\
\hline PI 489777 & 530,227 & 99,288 & 12,742 & 5 & 24 & 94 & 32 & 17,737 & 24 & 2,032 & 662,205 \\
\hline $\mathrm{Pb} 7$ & 169,234 & 18,338 & 2,800 & 2 & 6 & 37 & 11 & 3,010 & 7 & 1,245 & 194,690 \\
\hline Vijay & 138,673 & 16,769 & 2,715 & 2 & 6 & 33 & 17 & 2,929 & 3 & 1,343 & 162,490 \\
\hline WR 315 & 158,812 & 20,911 & 3,102 & 2 & 4 & 32 & 17 & 3,550 & 6 & 934 & 187,370 \\
\hline
\end{tabular}


genotypes and its putative function was Pentatricopeptide repeat-containing protein belonging to PPR family, PCMP-E subfamily. The PAVs result depicted that there were no genes deleted in ICCV 03312, IG 72953 and PI 489777 (Additional file 10). A maximum of 32 genes were found deleted in JG 62 followed by 30 genes in ICCV 00108.

\section{Miscellaneous variations}

In addition to above variations, an effort was made to identify miscellaneous variations like inter-chromosomal variations (CTX), intra-chromosomal variations (ITX) and inversions (INV). Of 539 miscellaneous variations, 339, 138 and 62 were CTX, ITX and INV respectively. To further avoid false positives, we have used stringent cutoff of 99. As a result 110 miscellaneous variations were identified on eight pseudomolecules (Table 2). CTX were in the range of $273 \mathrm{bp}$ to $667 \mathrm{bp}$ spread over Ca3 (22), Ca6 (16), Ca4 (10), Ca1 (7), Ca5 (7) and Ca7 (3). ITX were in the range of $86 \mathrm{bp}$ to $3.81 \mathrm{Mbp}$ spread over Ca2 (11), Ca4 (4), Ca5 (4), Ca7 (4), Ca8 (3) and Ca3 (2). While INV were in the range of $30 \mathrm{bp}$ to $4.76 \mathrm{Mbp}$ predominantly on Ca6 (8), followed by Ca4 (2), Ca7 (2) and $\mathrm{Ca} 2(1)$.

\section{Line specific variations}

A total of 164,856 unique line specific variations including 144,888 SNPs and 19,968 Indels were observed among 35 chickpea genotypes studied. Maximum number of line specific variations, 78,320, were observed in PI 489777 (68,799 SNPs and 9,521 Indels), and followed by 62,808 in IG 72953 (55,393 SNPs and 7,415 Indels) (Fig. 2a; Additional file 11). We further compared line specific variations among parental genotypes that segregate for abiotic stresses (like drought, salinity) and biotic stresses (like Fusarium wilt, Ascochyta blight, Botrytis grey mould). Although larger number of line specific SNPs and Indels were identified in the case of Helicoverpa resistant wild species genotype IG 72953, interestingly no species specific deletion and duplication of genes were identified (Fig. 2a). On contrary, in the case of parental genotypes of mapping population segregating

Table 2 Distribution of miscellaneous variations on eight pseudomolecules of chickpea

\begin{tabular}{lllllllllllll}
\hline \multirow{2}{*}{$\begin{array}{l}\text { Type of miscellaneous } \\
\text { variation* }\end{array}$} & \multicolumn{1}{l}{ Pseudomolecules } & & \multicolumn{1}{ll}{ Total } \\
\cline { 2 - 8 } & Ca1 & Ca2 & Ca3 & Ca4 & Ca5 & Ca6 & Ca7 & Ca8 & \\
\hline CTX & 4 & 2 & 2 & 4 & 9 & 10 & 30 & 6 & 67 \\
INV & 0 & 1 & 0 & 2 & 0 & 8 & 2 & 0 & 13 \\
ITX & 0 & 11 & 2 & 4 & 4 & 2 & 4 & 3 & 30 \\
Total & 4 & 14 & 4 & 10 & 13 & 20 & 36 & 9 & 110
\end{tabular}

${ }^{*} \mathrm{CTX}$ - inter-chromosomal translocations, ITX - intra-chromosomal, INV - inversions and translocation for Helicoverpa resistance (ICC $506 \times$ ICC 3137) the number of line specific SNPs and Indels differed significantly. Similarly, large number of line specific variations among parental lines of mapping populations segregating for Ascochyta blight (Fig. 2b), Fusarium wilt (Fig. 2c), Botrytis grey mould and salinity (Additional files 12 and 13), were identified that can be used for developing high density genetic maps, trait mapping as well as for marker-assisted selection. Among 35 chickpea genotypes, the line specific variations were $<100$ in case of ICC 1496, ICCV 00108, Pb 7, JG 130, ICC 4958, JAKI 9218 and $C$ 214. Among 35 genotypes, interestingly no line specific variation was observed in the case of $C 214$. Further, the number of line specific variations were in the range of $\sim 100$ to $\sim 5,000$ in case of remaining 26 genotypes (Additional file 13). The maximum percentage of line specific variations found in coding regions in ICC 1496 (21 \%) followed by ICCV 97105 (12 \%) (Additional file 14). The mean of the line specific variations in the coding region was found $6.4 \%$, while none of the line specific variations in coding regions were observed in the case of ICC 4958 and JAKI 9218. When the frame shift, start lost, stop gained and stop lost mutations were summed up and their percentage were calculated out of the total variations in coding regions, the maximum of $33.33 \%$ was observed in $\mathrm{Pb} 7$ genotype, while there were none in 8 genotypes (ICCV 04112, ICCV 04516, C 104, ICCV 00108, JG 11, JG 130, ICCV 10 and ICC 1431) (Additional file 15). Among the parental lines of MAGIC population, line specific SNPs, line specific deletions and line specific insertions were high in case of JG 11. Further, gene deletions ranged from 8 (JAKI 9218) to 30 (ICCV 00108), while gene duplications ranged from 17 (JG 130) to 1,120 (JAKI 9218) (Table 3). Overall large variation is evident at genome level in case of parental lines of MAGIC population. The main purpose of developing MAGIC populations is to create and harness the genetic diversity for crop improvement. In summary, the MAGIC lines developed from these lines will possess tremendous variation that can be used for allele mining and gene discovery. The line specific variants were further annotated using Uniprot repository. The annotation revealed the effect of these line specific variants on a number of transcription factors and their regulators like zinc finger protein, bHLH, WRKY, F-Box, bZIP, PHD, SCREAM and MADS box, etc. Along with TFs, disease resistace NB-LRR protein, heat shock proteins, DNA- damage repair proteins, nodulation signaling pathway related proteins were also affected (Additional file 16).

\section{Annotation of genome-wide variations}

In general, premature stop, frame-shift and presence/absence variations lead to genetic load by disabling the 

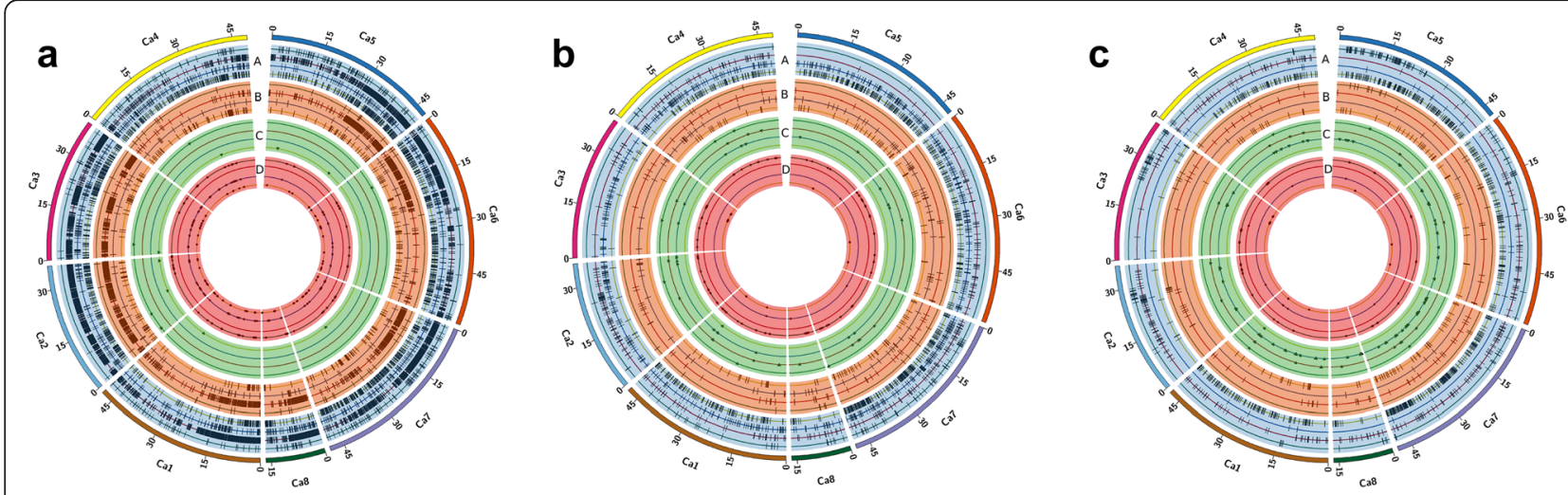

Fig. 2 Genome-wide variations identified in chickpea genotypes resistant or susceptible to important biotic stresses. Circos diagrams represent line specific SNPs, Indels, gene deletions and duplications. Each circos represents eight chickpea pseudomolecules and consists of four concentric rings where A represents SNPs, B represents Indels, C represents deletion and D represents duplication events. The deletion events are marked with triangles in green ring and circles represent duplications in red. a Distribution of variations among Helicoverpa resistant and susceptible genotypes. The green, red, blue, and yellow color inside all rings represents Vijay, IG 72953, ICC 506 and ICC 3137 respectively. b Distribution of variations among Fusarium wilt resistant and susceptible genotypes. The green, red, blue, and yellow color inside all rings represents C 104, JG 62, WR 315 and ICCV 05530 respectively. c Distribution of variations among Ascochyta blight resistant and susceptible genotypes. The green, red, blue, and yellow color inside all rings represents ICCV 04516, JG 62, Pb 7 and ICCV 05530 respectively

gene functions which may lead to inbreeding depression. Hence, we annotated the genome-wide variations. Among 2,351,154 variations, 38,078 were mis-sense, 352 were non-sense and 50,290 were the silent mutations (Additional file 17). However, large variations $(1,924,406)$ were in intergenic region. Of 419,262 variations detected in the genic regions, 328,171 were intronic variations and 91,091 were exonic variations (Additional file 18). Out of these exonic variations, 37,875 were nonsynonymous substitutions. On comparing the distribution of SNPs across genomic regions, SNPs were most abundant in intergenic regions $(81.85 \%)$ and the proportion of SNPs were high in the introns $(13.96 \%)$ than exons $(3.87 \%)$. Genetic variant annotation and effect prediction tool was used to predict the effect of all SNPs, homozygous SNPs and heterozygous SNPs identified among all 35 chickpea genotypes (Additional files 18, 19, and 20). The number of SNPs leading to stop-gain or non-sense mutations among different genotypes ranged from 18 (Ejerie) to 119 (IG 72953). The Indels were studied for their affects on the genome for each of the genotypes. Maximum insertions affecting the genic regions was seen in IG $72953(21.54 \%)$ while the least in ICCV 00108 (12.23 \%) (Additional file 21). Similarly, maximum deletions occurring in the genic regions were observed in IG $72953(21.16 \%)$ and the minimum of $12.90 \%$ in JAKI 9218 (Additional file 22).

We identified 373 variations in the "QTL-hotspot" region on $\mathrm{Ca} 4$, reported earlier to enhance the drought tolerance in chickpea [6,31] (Additional file 20). Among these variations, notably two codon insertions were found in Ca_04570 (present in "QTL-hotspot-b") belonging to $7 \mathrm{~S}$ seed storage gene family and reported to enhance seed size [32]. In addition we also identified 38 variations that were non-synonymous coding affecting a

Table 3 Line specific variations identified among parental lines of the chickpea MAGIC population

\begin{tabular}{|c|c|c|c|c|c|c|c|c|}
\hline Genotype & $\begin{array}{l}\text { Total } \\
\text { SNPs }\end{array}$ & $\begin{array}{l}\text { Line specific } \\
\text { SNPs }\end{array}$ & $\begin{array}{l}\text { Total } \\
\text { deletions }\end{array}$ & $\begin{array}{l}\text { Line specific } \\
\text { deletions }\end{array}$ & $\begin{array}{l}\text { Total } \\
\text { insertions }\end{array}$ & $\begin{array}{l}\text { Line specific } \\
\text { insertions }\end{array}$ & $\begin{array}{l}\text { Genes } \\
\text { deleted }\end{array}$ & $\begin{array}{l}\text { Genes } \\
\text { duplicated }\end{array}$ \\
\hline ICC 4958 & 340,803 & 3 & 29,976 & 1 & 28,429 & 0 & 23 & 47 \\
\hline ICCV 00108 & 125,680 & 49 & 9,217 & 6 & 8,907 & 2 & 30 & 323 \\
\hline ICCV 10 & 174,644 & 219 & 14,137 & 20 & 13,534 & 22 & 14 & 21 \\
\hline ICCV 97105 & 261,406 & 120 & 24,016 & 6 & 24,274 & 13 & 18 & 42 \\
\hline JAKI 9218 & 315,032 & 1 & 30,320 & 0 & 31,339 & 0 & 8 & 1,120 \\
\hline JG 11 & 207,785 & 496 & 17,690 & 36 & 17,640 & 31 & 21 & 25 \\
\hline JG 130 & 166,662 & 13 & 12,936 & 2 & 12,342 & 3 & 25 & 17 \\
\hline JG 16 & 147,615 & 95 & 10,579 & 10 & 10,094 & 6 & 27 & 73 \\
\hline
\end{tabular}


total of 17 genes with functions like heat stress transcription factor A-6b, EPF-like protein 4 and Early lightinduced protein (chloroplastic ELIP) (Additional file 23).

\section{Conclusion}

The genome-wide variations identified in the present study can be used for developing high density SNP arrays for genetics and breeding applications. Further, large number of line specific variations among the wild accessions indicate that the wild chickpea has much more diverse genepool than the cultivated chickpea, thus may contain useful genetic resources for chickpea improvement.

\section{Methods}

Plant material

Thirty five chickpea genotypes, used in the study, their pedigree, origin, market class and salient features are presented in Additional file 1.

\section{DNA isolation}

Genomic DNA was isolated from all 35 chickpea genotypes from 10-days old etiolated seedlings as described earlier [33]. The quality of DNA was checked on $0.8 \%$ agarose gel. The Qubit ${ }^{\circ}$ 2.0 Fluorometer (Life technologies, Thermo Fisher Scientific Inc. USA) was used for quantification of DNA.

\section{Library construction and sequencing}

Approximately, $1 \mu \mathrm{g}$ DNA from each sample was used for construction of a MiSeq sequencing library using TruSeq DNA sample Prep kit LT, (set A) FC-121-2001 (Illumina, San Diego, CA, USA) according to manufacturer's protocol. Briefly, column purified genomic DNA was sheared using Bioruptor ${ }^{\bullet}$ NGS (Diagenode, Belgium). The size of fragmented DNA was determined on $1.2 \%$ agarose gel and was found within the range of 200-1000 bp. After shearing, the end-repair was performed to convert the overhangs into blunt ends followed by adenylate 3' ends. Subsequently, indexed adaptors were ligated to the ends of the DNA fragments to make them ready for hybridization onto the flow cell.

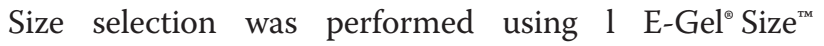
$2 \%$ agarose precast gels (Invitrogen) to get a target insert size of about $400 \mathrm{bp}$ and purified. PCRs (9 cycles) were performed to enriched the size selected DNA fragments having Illumina adapters on both the ends. The size distribution of amplified DNA library was checked on an Agilent Technologies 2100 bioanalyzer using Agilent high sensitivity DNA chip.

Denatured and diluted libraries were sequenced on Illumina MiSeq benchtop sequencer (Illumina, San Diego, CA, USA) using MiSeq Reagent Kit v2 (300-cycles) to generate 150 bases paired-end reads. Data was demultiplexed on the MiSeq instrument automatically and sample wise zipped FASTQ files were generated.

\section{Data filtering and alignment}

The raw data generated for each line were cleaned and trimmed using sickle version 1.200 (https://github.com/ najoshi/sickle). The cleaned data were aligned on to the reference chickpea genome [17] using Bowtie 2 [34]. The alignment data were further filtered to retain the reads mapped to only one region along the genome. The reads with a minimum mapping quality of 30 , were used for further analysis. Base Quality Score Recalibration (BQSR) and InDel Realignment components of The Genome Analysis Toolkit (GATK, v 3.1-1) [35], multiple utilities from Picard (v1.102) (http://broadinstitute.github.io/picard/) were used for post-processing of the bam files.

\section{Identification of genome-wide variations}

The alignment files generated after the above mentioned stringent criteria were used for variant discovery with GATK program. A position was reported as a variant for a genotype if the phred quality score $>30$ supported by a minimum read depth of 5 . Variants with less than $5 \mathrm{bp}$ flanking distance were filtered out. Distribution of DNA polymorphisms was assessed by calculating their frequency in a window size of $100 \mathrm{~Kb}$ along each pseudomolecule. For identification of effects of synonymous and non-synonymous SNPs and Indels, SnpEff program [36] was used. In-house Perl scripts were used to analyze the distribution of the variations (SNPs and Indels) across the genome. Line specific variations were reported only if the variation was present in only one genotype and the reference allele was present in rest of the genotypes. The line specific variations were further studied for their effects on the coding sequences and these variations were assigned GO IDs with the information retrieved from UniProtKB for the genes showing hits to UniProt IDs. Circos diagrams were used to plot line specific variations [37].

CNVnator was used to find the CNVs with an e-value cutoff of 1e-05 and results were annotated with genes $\geq$ $1,000 \mathrm{bp}$ in length [38]. False positives were eliminated by excluding the $\mathrm{CNVs}$ discovered by mapping the reads to CDC Frontier. PAVs were determined based on the sequencing depth $(<10 \%$ was considered as absence variation and $>50 \%$ was considered as presence variation). For determining miscellaneous variations like ITX, CTX and INV, paired-end reads from each sample were aligned to the reference genome (CDC Frontier) using Bowtie 2 with discordant flags and in end-to-end mapping mode. Picard (v1.102) (http://broadinstitute. github.io/picard/) was used to set the read group information on the alignment files and sorted by coordinate 
position using SAMtools (v0.1.19+) [39]. Breakdancer (v1.1.2) [40] was used to detect miscellaneous variations. The miscellaneous variations showing the presence of reads from control were omitted as they are considered as false positives. The miscellaneous variations were filtered by score equal to 99 thereby selecting a highly confident set of miscellaneous variations. Statistics of the miscellaneous variations were summarized by type in $\mathrm{R}$ (v3.1). Breakdancer provides only the breakpoint coordinates, within which the detected miscellaneous variations reside. So, in order to find the exact nucleotide coordinates of the miscellaneous variations, the reads supporting each of the miscellaneous variations were looked-up in the alignment files. This was performed using an inhouse tool in $\mathrm{C}++$.

\section{Availability of supporting data}

The data sets supporting the results of this article are available at http://ceg.icrisat.org/publicdomain.html.

\section{Additional files}

Additional file 1: Details on geographic origin, market class and pedigree information of 35 chickpea genotypes used in the study. (DOCX $17 \mathrm{~kb}$ )

Additional file 2: Summary of data generated for 35 chickpea genotypes and aligned to reference genome of chickpea (CDC Frontier). (DOC $70 \mathrm{~kb}$ )

Additional file 3: Distribution of SNPs, Indels and their effects in the chickpea genome (Ca1-Ca8). (DOCX $14 \mathrm{~kb}$ )

Additional file 4: Pairwise SNPs identified among 35 chickpea genotypes used in this study. (XLSX $17 \mathrm{~kb}$ )

Additional file 5: Homozygous and heterozygous SNPs identified in each chickpea genotype used in the study. Maximum heterozugous SNPs are evident in IG 72933 while homozygous SNPs in PI 489777. (TIF $309 \mathrm{~kb}$ )

Additional file 6: Summary of homozygous and heterozygous SNPs identified in 35 chickpea genotypes. (XLSX $11 \mathrm{~kb}$ )

Additional file 7: Summary of Indel lengths and their respective counts across the genome. (XLSX $9 \mathrm{~kb}$ )

Additional file 8: Summary of Indel ratio among 35 chickpea genotypes re-sequenced. (XLSX $12 \mathrm{~kb}$ )

Additional file 9: Gene duplications observed in 35 chickpea genotypes. (XLSX $2979 \mathrm{~kb})$

Additional file 10: Gene deletion detected in 35 chickpea genotypes used in this study. (XLSX $67 \mathrm{~kb}$ )

Additional file 11: Genome-wide variations identified in chickpea genotypes resistant or susceptible to Botrytis grey mould. Circos diagram represents line specific variations. Each circos represents eight chickpea pseudomolecules and consists of four concentric rings where $A$ represents SNPs, B represents Indels, C represents deletion and D represents duplication events. The

deletion events are marked with triangles in green ring and circles represent duplications in red. The green, red, blue, and yellow color inside all rings represents ICC 1496 (resistant), JG 62 (susceptible), ICCV 10 (susceptible), ICCV 05530 (resistant) respectively. (PNG 3357 kb)

Additional file 12: Genome-wide variations identified in chickpea genotypes tolerant or susceptible to salinity. Circos diagram represents line specific variations. Each circos represents eight chickpea pseudomolecules and consists of four concentric rings where $\mathrm{A}$ represents SNPs, B represents Indels, C represents deletion and D represents duplication events. The deletion events are marked with triangles in green ring and circles represent duplications in red. The green, red, blue, and yellow color inside all rings represents ICC 1431 (tolerant), JG 62 (tolerant), ICC 6263 (susceptible), JG 11 (tolerant) respectively. (PNG 3400 kb)

Additional file 13: Summary of line specific variations. (XLSX $11 \mathrm{~kb}$ ) Additional file 14: Summary of line specific variations in coding region identified in each chickpea genotype. (XLSX $12 \mathrm{~kb}$ )

Additional file 15: Summary of line specific variations leading to stop sites. (XLSX $11 \mathrm{~kb})$

Additional file 16: Gene ontology and their effects for the line specific variations in 35 genotypes. (XLSX $727 \mathrm{~kb}$ )

Additional file 17: Summary of all variant effects predicted by SNPEff. (XLSX $12 \mathrm{~kb}$ )

Additional file 18: Classification of all SNPs using SNPEff program. (XLSX $10 \mathrm{~kb}$ )

Additional file 19: Classification of heterozygous SNPs using SNPEff program in 35 chickpea genotypes. (XLSX $12 \mathrm{~kb}$ )

Additional file 20: Classification of homozygous SNPs using SNPEff program in 35 chickpea genotypes. (XLSX 12 kb)

Additional file 21: Summary of effects for the insertions. (DOCX $15 \mathrm{~kb}$ )

Additional file 22: Summay of effects for the deletions. (DOCX $15 \mathrm{~kb}$ ) Additional file 23: Summary of variations identified in the "QTL-hotspot" region reported by Varshney et al. [6, 8]. (XLSX $91 \mathrm{~kb})$

\section{Abbreviations}

WGRS: Whole genome re-sequencing; DEL: Deletions; INS: Insertions;

CTX: Inter-chromosomal translocations; ITX: Intra-chromosomal translocations; CNV: Copy number variations.

\section{Competing interests}

The authors declare that they have no competing interests.

\section{Authors' contributions}

RKV-Conceived the idea; MT, VK- Performed the experiments; AWK, AVSK, VG, MT - Analysed the data; PMG, SS- Contributed the germplasm lines for the study; RKV-Contributed reagents for the study; MT, RKV, AWK, MR- Wrote the manuscript; all authors read the MS and provided their consent. All authors read and approved the final manuscript.

\section{Authors' information}

Rajeev K Varshney is currently working as Research Program Director - Grain Legumes and Director - Center of Excellence in Genomics (http://

ceg.icrisat.org). He has extensive expertise in genomics and marker-assisted breeding. He led the efforts of sequencing two legume genomes (chickpea and pigeonpea) and contributed significantly to groundnut genome sequencing as well as several other genomes. Together with breeders at ICRISAT and other collaborating institutions he has led to the development of molecular breeding products in chickpea and groundnut.

Mahendar Thudi- is currently working as Scientist (Applied Genomics and Genotyping Services), he is heading the Genotyping Services Laboratory at ICRISAT, which aims at enhancing adoption of molecular tools in breeding programs for enhancing faster genetic gains. CEG offers SSR, DArT and SNP genotyping services to national partners as well as researches across the world. Aamir W. Khan is working as Scientific Officer in Computational Genomics. Vinay Kumar- a Special Project Scientist, applied genomics at ICRISAT. He is taking care of next generation sequencing facility at Center of Excellence in Genomics, ICRISAT. He has more than 5 years post doctoral experience in the field of genomics specially NGS applications, DNA testing, molecular markers genotyping and diversity analysis.

Pooran M. Gaur is Principal Scientist (Chickpea Breeding). He has 20 years experience in chickpea improvement.

Krishnamohan Katta is Special Project Scientist in Computational Genomics. Vanika Garg is a Research Scholar pursuing her PhD in Computational Genomics. 
Manish Roorkiwal is currently working as Scientist - Genomics and Molecular Breeding. He has a basic background in molecular genetics and applied genomics with more than 10 years of research experience. His research focuses on improving the crop productivity of legumes using next generation genomics and breeding approaches. Srinivasan Samineni is a chickpea breeder at ICRISAT.

\section{Declaration}

We greatly appreciate support from the CGIAR Research Program on Grain Legumes, ICRISAT, India, for publication of this article.

This article has been published as part of BMC Plant Biology Volume 16 Supplement 1, 2015: Selected articles from PlantGen 2015 conference: Plant biology. The full contents of the supplement are available online at http:// www.biomedcentral.com/bmcplantbiol/supplements/16/S1.

\section{Acknowledgements}

This work has been undertaken as part of the CGIAR Research Program on Grain Legumes, ICRISAT, India. ICRISAT is a member of CGIAR Consortium.

\section{Author details}

${ }^{1}$ International Crops Research Institute for the Semi-Arid Tropics (ICRISAT), Hyderabad, India. ${ }^{2}$ The University of Western Australia (UWA), Crawley, Western Australia, Australia.

\section{Published: 27 January 2016}

\section{References}

1. Singh KB. Chickpea breeding. In: Saxena MC, Singh KB, editors. The Chickpea. Wallingford: CAB International; 1987. p. 127-62.

2. Ladizinsky G, Adler A. The origin of chickpea Cicer arietinum L. Euphytica. 1976;25:211-7

3. Van der Maesen LJG. Cicer L. Origin, history and taxonomy of chickpea. In: Saxena MC, Singh KB, editors. The Chickpea. Aberystwyth: CAB International; 1987. p. 11-34.

4. FAOSTAT (2013) http://faostat.fao.org/site/339/default.aspx. Accessed on July 15,2015

5. Roorkiwal M, Nayak SN, Thudi M, Upadhyaya HD, Brunel D, Mournet $P$, et al. Allele diversity for abiotic stress responsive candidate genes in chickpea reference set using gene based SNP markers. Front Plant Sci. 2014;5:248.

6. Varshney RK, Thudi M, Nayak SN, Gaur PM, Kashiwagi J, Krishnamurthy L, et al. Genetic dissection of drought tolerance in chickpea (Cicer arietinum L.). Theor Appl Genet. 2014;127:445-62.

7. Thudi M, Gaur PM, Krishnamurthy L, Mir RR, Kudapa H, Fikre A, et al. Genomics-assisted breeding for drought tolerance: a dream comes true in chickpea! Funct Plant Biol. 2014:41:1178-90.

8. Varshney RK, Terauchi R, McCouch SR. Harvesting the promising fruits of genomics: applying genome sequencing technologies to crop breeding. PLOS Biol. 2014;12(6):e1001883.

9. $3 K$ RGP. The 3,000 rice genomes project. GigaScience 2014;3:7.

10. Jiao $Y$, Zhao H, Ren L, Song W, Zeng B, Guo J, et al. Genome-wide genetic changes during modern breeding of maize. Nat Genet. 2012;44:812-5.

11. Mace ES, Tai S, Gilding EK, Li Y, Prentis PJ, Bian L et al. Whole-genome sequencing reveals untapped genetic potential in Africa's indigenous cereal crop sorghum. Nat Commun. 2013:4: doi:10.1038/ncomms3320.

12. Saxena RK, Edwards D, Varshney RK. Structural variation in plant genomes. Brief Funct Genomics. 2014; doi:10.1093/bfgp/elu016.

13. Wang W, Wang S, Hou C, Xing Y, Cao J, Wu K, et al. Genome-wide detection of copy number variations among diverse horse breeds by Array CGH. PLoS One. 2014;9(1):e86860.

14. Brohammer A. Genic copy number variation associated with abiotic/biotic stress among a set of progenitors of contemporary maize germplasm. https://www.ideals.illinois.edu/handle/2142/49803 2014.

15. Muñoz-Amatriaín M, Eichten SR, Wicker T, Richmond TA, Mascher M Steuernagel B, et al. Distribution, functional impact, and origin mechanisms of copy number variation in the barley genome. Genome Biol. 2013;14:R58.

16. Michael TP, Jackson S. The first 50 plant genomes. The Plant Genome. 2013;6(2)

17. Varshney RK, Song C, Saxena RK, Azam S, Yu S, Sharpe AG, et al. Draft genome sequence of chickpea (Cicer arietinum) provides a resource for trait improvement. Nat Biotechnol. 2013;31:240-6.
18. Deokar AA, Ramsay L, Sharpe AG, Diapari M, Sindhu A, Bett K, et al. Genome wide SNP identification in chickpea for use in development of a high density genetic map and improvement of chickpea reference genome assembly. BMC Genomics. 2014;15:708.

19. Hiremath PJ, Farmer A, Cannon SB, Woodward J, Kudapa H, Tuteja R, et al. Large-scale transcriptome analysis in chickpea (Cicer arietinum L.), an orphan legume crop of the semi-arid tropics of Asia and Africa. Plant Biotechnol J. 2011;9:922-31.

20. Gujaria N, Kumar A, Dauthal P, Dubey A, Hiremath P, Bhanu Prakash A, et al. Development and use of genic molecular markers (GMMs) for construction of a transcript map of chickpea (Cicer arietinum L.). Theor Appl Genet. 2011;122:1577-89.

21. Varshney RK, Hiremath PJ, Lekha PT, Kashiwagi J, Balaji J, Deokar AA, et al. A comprehensive resource of drought- and salinity- responsive ESTs for gene discovery and marker development in chickpea (Cicer arietinum L.). BMC Genomics. 2009;10:523.

22. Zhou Z, Jiang Y, Wang Z, Gou Z, Lyu j, Li W et al. Resequencing 302 wild and cultivated accessions identifies genes related to domestication and improvement in soybean. Nat. Biotechnol. 2014; 33: doi:10.1038/nbt.3096.

23. Xu X, Liu X, Ge S, Jensen JD, Hu F, Li X, et al. Resequencing 50 accessions of cultivated and wild rice yields markers for identifying agronomically important genes. Nat Biotechnol. 2012;30:105-11.

24. Qina C, Yub C, Shena Y, Fang X, Chenb L, Min J, et al. Whole-genome sequencing of cultivated and wild peppers provides insights into Capsicum domestication and specialization. Proc Natl Acad Sci. 2014;111:5135-40.

25. Xu J, Yuan Y, Xu Y, Zhang G, Guo X, Wu F, et al. Identification of candidate genes for drought tolerance by whole-genome resequencing in maize. BMC Plant Biol. 2014;14:83.

26. The 100 Tomato Genome Sequencing Consortium, Aflitos S, Schijlen E, de Jong $\mathrm{H}$, de Ridder $\mathrm{D}$, Smit $\mathrm{S}$, et al. Exploring genetic variation in the tomato (Solanum section Lycopersicon) clade by whole-genome sequencing. Plant J. 2014;80:136-48.

27. Doddamani D, Khan AW, Katta MAVSK, Agarwal G, Thudi M, Ruperao P, et al. CicArVarDB: SNP and InDel database for advancing genetics research and breeding applications in chickpea. Database. 2015;1-7. doi: 10.1093/database/ bav078.

28. Kujur A, Bajaj D, Upadhyaya HD, Das S, Ranjan R, Shree T, et al. Employing genome-wide SNP discovery and genotyping strategy to extrapolate the natural allelic diversity and domestication patterns in chickpea. Front Plant Sci. 2015;6:162.

29. Hiremath PJ, Kumar A, Penmetsa RV, Farmer A, Schlueter JA, Chamarthi SK, et al. Large-scale development of cost-effective SNP marker assays for diversity assessment and genetic mapping in chickpea and comparative mapping in legumes. Plant Biotechnol J. 2012;10:716-32.

30. Gaur R, Azam S, Jeena G, Khan AW, Choudhary S, Jain M, Yadav G, Tyagi AK, Chattopadhyay D, S Bhatia S. High-throughput SNP discovery and genotyping for constructing a saturated linkage map of chickpea (Cicer arietinum L.). DNA Res. 2012:19:357-373.

31. Varshney RK, Gaur PM, Chamarthi SK, Krishnamurthy L, Tripathi S, Kashiwagi J et al. Fast-track introgression of "QTL-hotspot" for root traits and other drought tolerance traits in JG 11, an elite and leading variety of chickpea. Plant Genome. 2013:6(3). doi:10.3835/plantgen ome2013.07.0022.

32. Kale SM, Jaganathan D, Ruperao P, Chen C, Punna R, Kudapa $\mathrm{H}$ et al. Prioritization of candidate genes in "OTL-hotspot" region for drought tolerance in chickpea (Cicer arietinum L.). Sci Rep 2015;5:15296.

33. Cuc LM, Mace E, Crouch J, Quang VD, Long TD, Varshney RK. Isolation and characterization of novel microsatellite markers and their application for diversity assessment in cultivated groundnut (Arachis hypogaea). BMC Plant Biol. 2008:8:55

34. Langmead B, Salzberg SL. Fast gapped-read alignment with Bowtie 2. Nat Methods. 2012;9(4):357-9. http://doi.org/10.1038/nmeth.1923.

35. McKenna A, Hanna M, Banks E, Sivachenko A, Cibulskis K, Kernytsky A et al. The genome analysis toolkit: a MapReduce framework for analyzing next-generation DNA sequencing data. Genome Res. 2010; 20(9):1297-303

36. Cingolani P, Platts A, Wang le L, Coon M, Nguyen T, Wang $L$, et al. A program for annotating and predicting the effects of single nucleotide polymorphisms, SnpEff: SNPs in the genome of Drosophila melanogaster strain w1118; iso-2; iso-3. Fly (Austin). 2012:6 Suppl 2:80-92.

37. Krzywinski M, Schein J, Birol I, Connors J, Gascoyne R, Horsman D, et al. Circos: an information aesthetic for comparative genomics. Genome Res. 2009;19:1639-45. 
38. Li H, Handsaker B, Wysoker A, Fennell T, Ruan J, Homer N, et al. The Sequence alignment/map (SAM) format and SAMtools. Bioinformatics. 2009; 25:2078-9.

39. Abyzov A, Urban AE, Snyder M, Gerstein M. CNVnator: an approach to discover, genotype, and characterize typical and atypical CNVs from family and population genome sequencing. Genome Res. 2011;21:974-84.

40. Fan X, Abbott TE, Larson D, Chen K. BreakDancer - Identification of genomic structural variation from paired-end read mapping. Curr Protoc Bioinformatics. 2014. doi: 10.1002/0471250953.bi1506s45.

Submit your next manuscript to BioMed Central and take full advantage of:

- Convenient online submission

- Thorough peer review

- No space constraints or color figure charges

- Immediate publication on acceptance

- Inclusion in PubMed, CAS, Scopus and Google Scholar

- Research which is freely available for redistribution

Submit your manuscript at www.biomedcentral.com/submit 